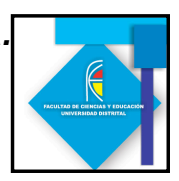

\title{
A PROPÓSITO DE LA FORMALIZACIÓN DE LOS FENÓMENOS FÍSICOS: UN ANÁLISIS DESDE LA PERSPECTIVA NEWTONIANA
}

\section{A PURPOSE OF THE FORMALIZATION OF THE PHYSICAL PHENOMENA: A PERSPECTIVE FROM NEWTONIAN ANALYSIS}

\author{
Andrés Ospina Ramírez ${ }^{1}$ \\ Érika Tobón Cardona ${ }^{2}$ \\ Yirsen Aguilar Mosquera ${ }^{3}$
}

\begin{abstract}
Resumen
El estudio de los procesos de formalización de los fenómenos físicos arroja pautas para la enseñanza de las ciencias, permite de algún modo esclarecer la relación físia-matemática y el papel de la experimentación en el aprendizaje de las ciencias. En este sentido se plantea que, el análisis de los procesos de formalización de la teoría del color y la propagación de la luz llevados a cabo por Newton y algunos estudiantes en contextos socioculturales particulares, permite no solo explicitar tal relación sino que permite el planteamiento de alternativas didácticas en la enseñanza de la física. Como propuesta se realiza una caracterización de los procesos de formalización en donde se destaca la organización de experiencias, la caracterización y cuantificación, el papel de la experimentación, la matematización, y los procesos de socialización. Se contrasta la manera de pensar y formalizar el fenómeno de la luz y el color desarrollada por Newton y algunos estudiantes, a partir de lo cual se teoriza y se crea una ruta para la formalización de los fenómenos físicos. Finalmente, como alternativa para la enseñanza, con estos componentes se configura una propuesta de intervención en el aula, donde el objetivo no es adquirir un cúmulo de conocimientos científicos sino desarrollar procesos de formalización en los estudiantes y docentes a partir de acciones concretas como: diseñar métodos de medición, identificar atributos, representar, relacionar variables, confrontar resultados, deducir leyes, confrontar ideas, entre otras.
\end{abstract}

Palabras clave: Formalización, experimentación, experiencia, caracterización, cuantificación, matemáticas, física, historia, epistemología, ciencia, fenomenología, educación.

\footnotetext{
${ }^{1}$ Estudios Culturales sobre las CienCias y su EnSEÑanZA -ECCE-Facultad de Educación, Universidad de Antioquia. Medellín, Colombia. rolo246@hotmail.com

${ }^{2}$ Estudios CUlTURAles SOBRE LAS CienCiAS y Su ENSEÑANZA -ECCE-Facultad de Educación, Universidad de Antioquia. Medellín, Colombia. eudes2511@yahoo.es

${ }^{3}$ Estudios Culturales SOBRe LAS CienCiAs y Su EnSEÑANZA -ECCE-Facultad de Educación, Universidad de Antioquia. Medellín, Colombia.yirsena@yahoo.es
} 
$5^{\circ}$ CONGRESO NACIONAL DE ENSEÑANZA DE LA FÍSICA.

Universidad Pedagógica Nacional (U. P. N)

Universidad Distrital Francisco José de Caldas (U. D. F. J. C)

Bogotá, Colombia. 16 al 20 de mayo 2011

\section{Abstract}

The study of the processes of formalization of physical phenomena yields guidelines for science education, can somehow clarify the physical-mathematical relationship and the role of experimentation in science learning. In this regard it is argued that the analysis of the processes of formalization of the theory of color and light propagation carried out by Newton and some students in particular socio-cultural contexts, can not only explain such a relationship but allows the approach educational alternatives in the teaching of physics. As a proposal is made a characterization of the formalization process which highlights the organization of experience, characterization and quantification, the role of experimentation, mathematization, and the processes of socialization. It contrasts the thinking and formalize the phenomenon of light and color developed by Newton and some students, from which it is theorized and creating a path for the formalization of physical phenomena. Finally, as an alternative to teaching, these components are configured with a proposal for intervention in the classroom, where the goal is not to acquire a wealth of scientific knowledge but to develop formalized processes for students and teachers from specific actions such as: design measurement methods, identify attributes, perform, link variables, compare results, deduce laws, compare ideas, among others.

Keywords: Formalization, experimentation, experience, characterization, quantification, mathematics, physics, history, epistemology, science, phenomenology, education.

\section{Relación matemática-física en la enseñanza de las ciencias}

La Ciencia, más allá de los posibles significados que las distintas disciplinas le otorguen, es una herramienta humana que ha facilitado la comprensión del mundo, de la naturaleza y de los humanos como intérpretes del universo. Ahora bien, cosa distinta es la enseñanza de las ciencias puesto que viene determinada por múltiples factores como: la noción de ciencia, la selección de contenidos, los objetivos, la cultura, la familia, la interdisciplinariedad, entre otros; éstos parecen desfigurar esa intención del docente de conocerse a través de la investigación ${ }^{4}$ y constituyen una idea de ciencia y de hombre de ciencia ajena a toda realidad interpretativa.

Esto se observa en muchas prácticas de enseñanza donde se evidencia, en docentes y alumnos, una tendencia a matematizar la física confundiéndola con la mera aplicación de fórmulas y algoritmos a los conceptos y las teorías físicas, mientras que otros se inclinan por una enseñanza y aprendizaje de los conceptos y teorías físicas que no involucran el uso de formalismos matemáticos. En estas distintas maneras de enseñar y de aprender subyacen distintos significados de conceptos propios de la enseñanza como ciencia, realidad, aprendizaje y evaluación, es decir, la relación matemática-física establecida por estudiantes y docentes obedece a formas particulares de considerar los elementos que intervienen en ella. El estudio de los procesos de formalización permite una aproximación al sistema que conecta los anteriores elementos y que configura la idea de ciencia, concepto que determina las posibles maneras de enseñar y aprender la física, estas a su

\footnotetext{
${ }^{4}$ Lo anterior evidencia en la Ciencia no sólo una forma de predecir los fenómenos sino también una forma de conocer a los científicos y a la cultura en la cual existieron. Esta reflexión es producto de las discusiones adelantadas en el seminario de práctica docente: Integración Didáctica VIII (2009-2) de la Licenciatura en Matemáticas y Física, Universidad de Antioquia.
} 


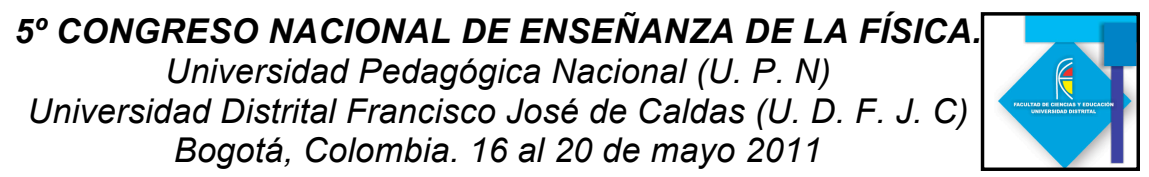

vez se pueden comprender con la intención de razonar sobre las particulares formas de interpretar el mundo.

Ahora bien, es necesario caracterizar los procesos de formalización a partir del estudio de un fenómeno en particular e indagar por la relación matemática-física a través de dichos procesos. Además, se deben analizar las consecuencias pedagógicas del estudio de la formalización de los fenómenos físicos.

Atendiendo a lo anterior, se realiza un estudio de caso en el que se aborda la teoría de Newton sobre la luz y el color, además de las formas explicativas que dan algunos estudiantes en su intensión de formalizar el fenómeno de la propagación de la luz.

Del estudio realizado se deducen las características de los procesos de formalización de los fenómenos físicos.

\section{Características de los procesos de formalización}

\section{Organización de experiencias}

Se compilan las experiencias frente a lo conocido del fenómeno, es decir, se recogen todos los conocimientos adquiridos hasta el momento sobre el mismo. Se observa en los primeros axiomas de la obra de Newton de lo que se conocía de la propagación de la luz.

\section{El papel de la experiencia en el proceso de formalización}

Permite orientar la visualización de un fenómeno, es decir la percepción de los objetos por medio de los sentidos y los conocimientos desarrollados. Algunas experiencias son explicitas en los axiomas planteados por Newton, las cuales se traducen en un resumen de lo que hasta la época se había tratado en Óptica, aquí las experiencias derivan de la adquisición de conocimientos concretos, pero no solamente se adquieren de esta manera, sino de las múltiples relaciones que el sujeto establece con el medio cultural y social. De este modo, las experiencias se refieren a un compendio de conocimientos que pueden ser de dos tipos: uno se encuentra relacionado con aquellas experiencias donde el sujeto que las vive puede argumentar razones de su procedencia; en el otro se encuentran aquellas experiencias las cuales no se les puede atribuir una causa. Las experiencias son adquiridas en el tiempo y en un contexto determinado, estas se pueden organizar y utilizar en situaciones nuevas, donde el sujeto sienta la necesidad de comprender y explicar algo que le inquieta en su vida. Para el caso de Newton, fue la necesidad de comprender y explicar la descomposición de la luz mediante una relación físico-matemática.

\section{Experiencias concretas}

Son aquellas experiencias que se hacen explicitas al momento de identificar su procedencia y el conocimiento utilizado, un ejemplo de ello son los axiomas planteados por Newton, los cuales se traducen en un resumen de lo que hasta la época se había tratado en Óptica, aquí las experiencias derivan de la adquisición de conocimientos socialmente aceptados. 


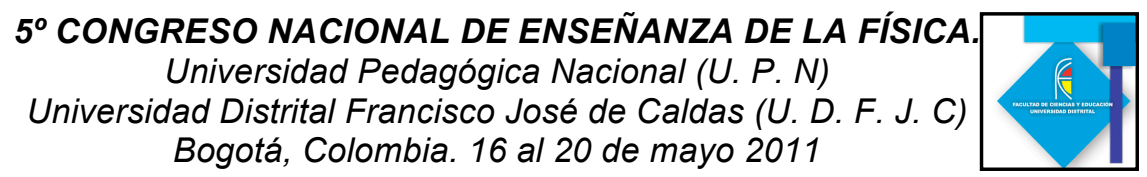

\section{Experiencias complejas}

Existen una serie de experiencias cuya procedencia es desconocida, parecen ser fruto de las múltiples relaciones que el sujeto establece con el medio cultural, social y consigo mismo. Un ejemplo de ello, es la forma cómo Newton pensó y construyó los diferentes experimentos para explicar y comprender la dispersión de la luz. No se puede tener certeza de la experiencia concreta que le permitió llevar a cabo los procesos experimentales, es decir, cómo saber, por ejemplo, la experiencia que le permitió a Newton desarrollar el primer experimento, que no fue referido a los colores de las luz, sino a los colores naturales para determinar los grados de refrangibilidad cuando son vistos a través de un prisma. La experiencia de la cual hace uso en este caso no es explícita, es decir, no hay información clara de su procedencia y el conocimiento utilizado.

\section{Caracterización y cuantificación}

En esta parte a los atributos o cualidades del fenómeno les es otorgada una forma cualitativa o cuantitativa. Así pues, se crea la posibilidad de cuantificar algunas de las características, atributos o cualidades identificadas a través de la experiencia cuando se establece, por ejemplo, una posible relación entre los rayos, su grado de refrangibilidad y los colores que aparecen.

\section{Representación}

Es un producto de las relaciones entre la actividad del sujeto con un objeto (Por objeto se significa una serie distinta de fenómenos, cosas, hechos, etc.), donde se codifica la percepción de estos en imágenes mentales que son plasmadas mediante iconos, símbolos, insignias de carácter gráfico. Un ejemplo claro lo constituye los diferentes dibujos que elaboró Newton en su obra, los cuales permitieron comprender y explicar no solo el fenómeno de la luz sino el diseño de los diferentes experimentos. Es de entender así que la representación, como elaboración mental, es una construcción del sujeto como medio de aproximación a una realidad subjetiva que permite facilitar la descripción de los objetos y la formulación de preguntas.

\section{Cuantificación}

El sujeto reconoce algunas nociones físicas cuando establece una posible relación entre conceptos, esto permite crear la posibilidad de cuantificar algunas características, atributos o cualidades de estos. Un ejemplo es el trabajo que Newton hace al establecer un isomorfismo entre rayo y línea recta, atribuyéndole una forma a la propagación de la luz que le permite establecer relaciones matemáticas entre los grados de refrangibilidad (ángulo de desviación de los rayos) para caracterizar la formación de los colores; es así, como la luz de color azul es más refrangible que la luz de color rojo. Dichos grados de refrangibilidad, para los diferentes rayos de la luz de sol, se convierten en una magnitud susceptible de ser medible, pues se crea la posibilidad de asociar un color a un rayo de luz. 


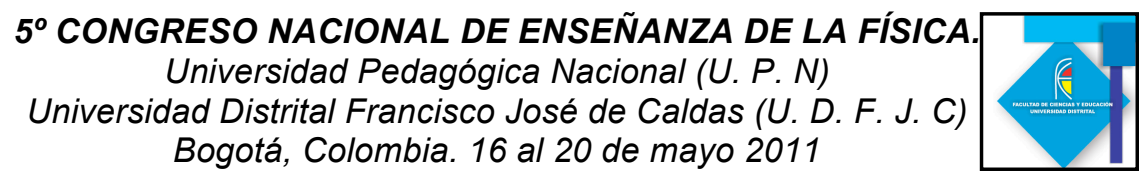

\section{Experimentación}

La experimentación es entendida como todo proceso intelectual y tangible en el cual se pone en juego el comportamiento de las características, atributos o cualidades de un fenómeno con el objetivo de encontrar relaciones entre ellos o relaciones no conscientes, es decir, nuevos conocimientos. Se pone de manifiesto en aspectos concretos y conscientes en la búsqueda de la dependencia entre los rayos homogéneos y sus grados de refrangibilidad y de la independencia del espectro de colores con respecto al medio (prisma).

\section{Experimentación mental}

Es una actividad del pensamiento que involucra procesos imaginativos donde se pone a actuar, bajo distintas circunstancias, objetos, contextos o situaciones específicas. Los objetos (por objeto se significa una serie distinta de fenómenos, cosas, hechos, etc.) pueden ser distinguidos, reconocidos por la experiencia mediante relaciones análogas, para hacer alguna predicción de lo que ocurriría cuando interactúan las características, atributos o cualidades de estos objetos. Todos estos elementos se presentan en una situación dada donde se busca identificar cambios relevantes de los objetos. En este proceso, se examinan ciertos cambios (acontecimientos relevantes) que son controlados por la persona, control que parte de una labor mental de experimentar lo observado sobre lo conocido, de tal forma que estos concuerden con la experiencia, dándoles una posibilidad de existencia. Por otro lado, la experimentación mental antecede a la experimentación tangible, esto se refleja en los montajes experimentales que hace Newton, pues obedecen a una planeación mental donde se piensa la disposición de ciertos objetos como el rayo de luz, el prisma, una habitación oscura, el espectro de colores, entre otros, antes de elaborar toda una serie de montajes experimentales para dar cuenta de las relaciones existentes entre los objetos, de tal forma que piensa como controlar la desviación de los rayos, la interferencia del prisma, el orificio en la pared y demás, para explicar el espectro de colores.

Toda actividad experimental obedece a una experiencia concreta o compleja que se desarrolla con la finalidad de construir conocimiento.

\section{Experimentación tangible}

Es toda actividad experimental donde la experimentación mental es contrastada con la manipulación directa de objetos. Newton utiliza todas sus experiencias (complejas y concretas) en una experimentación de este tipo, pues retoma los objetos pensados para manipularlos directamente, como es el caso de la posición del prisma, la amplitud del espectro de colores, la dirección del rayo de luz cuando penetra dentro de la habitación y demás.

\section{Matematización}

Se da juntamente con la cuantificación y búsqueda de relaciones entre las características del fenómeno. En este punto, el objetivo es hallar relaciones matemáticas entre las características cuantificables, además poner en juego la relación de dependencia de estos atributos, esta descripción puede ser estrictamente cualitativa. La matematización se pone 
de manifiesto en aspectos concretos y conscientes de la búsqueda de dependencia entre los rayos homogéneos y sus grados de refrangibilidad. La composición de la luz blanca en los diferentes colores es independiente de las propiedades del prisma.

\section{El papel de las matemáticas en la formalización}

La matematización es el resultado de la cuantificación de variables dada en la experimentación donde se pueden construir modelos matemáticos. La asignación de modelos matemáticos hecha por Newton da cuenta del nivel de conocimiento que él tenía de la matemática, porque manejando la geometría y la trigonometría de su tiempo pudo darle forma al fenómeno de la luz. Por tanto la complejidad de los modelos matemáticos asignados a los resultados experimentales obedece a los conocimientos que posee el sujeto sobre matemáticas. Recíprocamente, en ocasiones es necesario crear nuevos objetos matemáticos para poder representar alguna característica observada en el fenómeno. Al respecto ¿podría considerarse el objeto físico rayo un objeto matemático? En los planteamientos de Newton sí, puesto que las fronteras entre física y matemática son allí indistinguibles, no obstante el rayo como objeto físico posee una gran similitud con el concepto vector como objeto matemático. Ahora bien, todo sujeto hace uso de la experiencia para la construcción de conocimiento; por ende el saber que se utiliza para este fin es tanto individual como colectivo pues de algún modo es parte del conocimiento que culturalmente se ha constituido. En el origen como en el fin de la creación del concepto rayo se encuentra todo el saber de Newton, saber que comprende disciplinas como la filosofía, la geometría, la alquimia, la teología, entre otras. Ninguno de estos conocimientos puede ser nombrado como el responsable de la creación del concepto rayo, son todos en conjunto; son la matemática y la física indistinguibles en este punto, de tal modo que la matematización de los fenómenos físicos no puede considerarse parte de la matemática o parte de la física únicamente.

\section{Socialización}

Comprende la discusión con otros de la forma otorgada a un fenómeno con la intención de ser validada o reformada. Newton da muestra de socializar su obra para ser validada y reconfigurada no como una opción de construir conocimiento científico sino de mejorar la forma dada a la luz. Al respecto, Newton hacia hipótesis relativas, por ejemplo, al carácter corpuscular de la luz, pero negaba que dichas hipótesis fueran la base de su constructo óptico, en particular su teoría de los colores; y fue la incomprensión de este hecho por parte de sus colegas (Hooke, Pardies, Huygens), lo que llevó a Newton a mejorar su tratado sobre la luz y los colores publicado en 1704.

En el siguiente esquema se representan las relaciones entre las características de los procesos de formalización revelando su naturaleza compleja que no permite un orden determinado. 
$5^{\circ}$ CONGRESO NACIONAL DE ENSEÑANZA DE LA FÍSICA

Universidad Pedagógica Nacional (U. P. N)

Universidad Distrital Francisco José de Caldas (U. D. F. J. C)

Bogotá, Colombia. 16 al 20 de mayo 2011

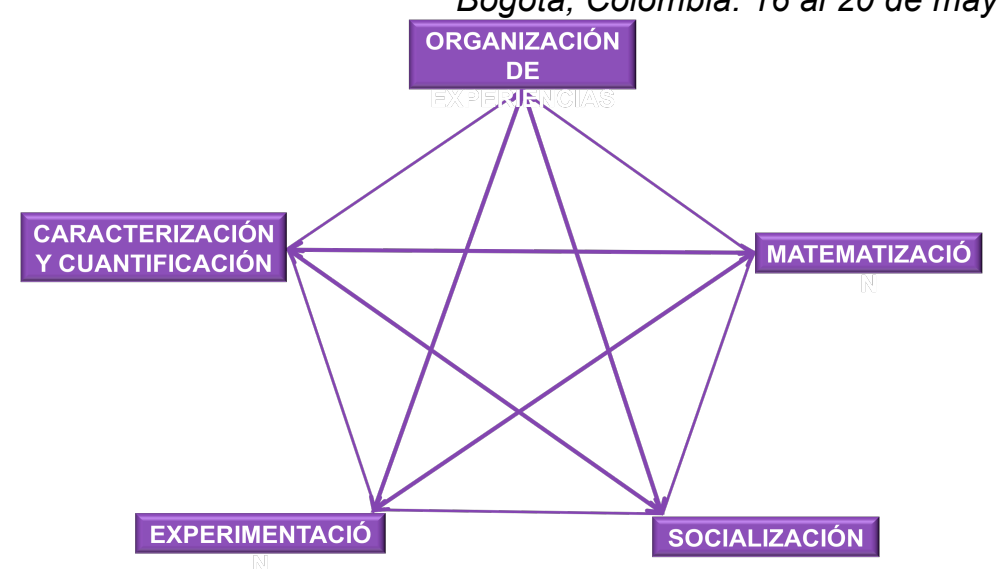

\section{Acciones asociadas a los procesos de formalización}

Las características de los procesos de formalización se relacionan en forma compleja en dichos procesos, puesto que no existen fronteras entre una y otra. De esta manera, cuando se experimenta, por ejemplo, la organización de las experiencias, la caracterización, matematización y socialización están inmiscuidas en el mismo proceso. Empero, se pueden definir algunas acciones específicas que transversalizan los procesos de formalización y que puede desarrollar cada sujeto en su intención de darle forma a un fenómeno, estas son: medir, manipular, diseñar métodos de medición, representar, observar, reflexionar, relacionar variables, deducir leyes, identificar atributos, contrastar resultados e ideas, entre otras. No puede considerarse que una de las acciones corresponda, exclusivamente, a un aspecto de los procesos de formalización. Reflexionar, por ejemplo, es una acción necesaria para organizar experiencias, experimentar, caracterizar, entre otras. A continuación se observa una tabla con las acciones y sus significados.

\begin{tabular}{|l|l|}
\hline \multicolumn{1}{|c|}{ ACCIONES } & \multicolumn{1}{c|}{ SIGNIFICADO } \\
\hline Manipular & Disponer de materiales para utilizarlos con un fin determinado. \\
\hline $\begin{array}{l}\text { Diseñar métodos de } \\
\text { medición }\end{array}$ & Planear estrategias para comparar magnitudes \\
\hline Medir & $\begin{array}{l}\text { Comparar magnitudes, asignar valores a los atributos identificados (pueden ser } \\
\text { cualitativos o cuantitativos). }\end{array}$ \\
\hline Observar & $\begin{array}{l}\text { Poner en contacto al sujeto con los objetos, con la finalidad de examinarlos } \\
\text { detenidamente. }\end{array}$ \\
\hline Describir & Narrar las características de los objetos. \\
\hline Identificar atributos & Encontrar las cualidades de un objeto. \\
\hline Representar & Modelar un objeto para abstraer los atributos mediante una construcción icónica. \\
\hline Relacionar variables & Asociar atributos de acuerdo a la independencia o dependencia que se da entre ellos. \\
\hline Confrontar resultados & Establecer diferencias o similitudes entre los análisis asociados a experimentos. \\
\hline Deducir leyes & $\begin{array}{l}\text { Enunciar una oración compuesta en la que se generaliza el comportamiento de los } \\
\text { atributos de un objeto. }\end{array}$ \\
\hline Confrontar ideas & $\begin{array}{l}\text { Cuestionar los argumentos del otro para llegar a acuerdos sobre formas de } \\
\text { comprensión, métodos, técnicas, etc. }\end{array}$ \\
\hline Reflexionar & $\begin{array}{l}\text { Retomar ideas o pensamientos anteriores para detenerse en ellos y dar un sentido a lo } \\
\text { considerado. }\end{array}$ \\
\hline
\end{tabular}




\section{Los procesos de formalización: un objetivo pedagógico}

La anterior caracterización del proceso de formalización le otorga una función no tradicional al docente de ciencias. El objetivo no es transmitir ciencia considerándola como el cúmulo de conocimientos ya elaborados, sino construir conocimiento favoreciendo el enriquecimiento del proceso de formalización de los fenómenos, mostrando la ciencia como un proceso dinámico en permanente construcción.

La labor del maestro es disponer espacios donde se organicen las experiencias, se caractericen y cuantifiquen los fenómenos, donde se experimente, donde se matematice, donde se socialicen las formas otorgadas a un fenómeno. El docente sería un exponente del conocimiento científico. La función del maestro y del alumno, en un contexto social, diverso y complejo, es la de convencer a los demás de que la forma como se entiende un fenómeno puede ser la mejor, la más completa. Por tanto, el maestro de ciencias debe fomentar el aprendizaje de las matemáticas, pero al interior de la física, mostrándola como parte de la física misma, ya que la matematización de los fenómenos le otorga validez a los mismos.

\section{Algunas conclusiones}

Se identifican los componentes de los procesos de formalización, estos son: organización de experiencias, caracterización y cuantificación, experimentación, matematización y socialización. Al interior de los diferentes componentes 0 características de los procesos se aprecian algunos aspectos relevantes de los mismos como el papel de la cuantificación y la representación en la caracterización de los objetos, las experiencias complejas y concretas en la organización de la experiencia y el papel de la experimentación mental y tangible en la experimentación. Los componentes de los procesos de la formalización de los fenómenos físicos configuran un entramado en forma de red que conecta cada característica en un sistema de relaciones complejas.

Los procesos de formalización pueden ser manifestados por aquellos que piensan los fenómenos físicos. Estos procesos se pueden enriquecer favoreciendo ciertas condiciones. En ese sentido, importa el contexto y los conocimientos culturales adquiridos en una época en particular. Newton logra formalizar el color y la propagación de la luz gracias a sus estudios y al contexto científico en el que se desenvuelve, de igual manera, algunas experiencias de los estudiantes, el nivel y contexto educativo son un factor determinante al momento de formalizar los fenómenos físicos.

Dentro de los procesos de formalización, la socialización resulta ser un componente relevante, pues permite confrontar ideas y discutir los métodos de medición en los estudiantes; además de refinar los argumentos. Se da oportunidad al disentimiento y fortalecimiento de los argumentos o ideas propias.

A nivel de la enseñanza de la física, la matematización es un componente poco explorado en los procesos de formalización de aquellos que dan forma a un fenómeno, esto viene dado por el requerimiento de un mayor nivel de apropiación de ciertos conceptos y de las relaciones entre los atributos de un objeto, para asociar o construir una forma o modelo matemático que dé cuenta de la relación entre la cuantificación de dichos atributos y su comportamiento futuro. Sin embargo, no se 


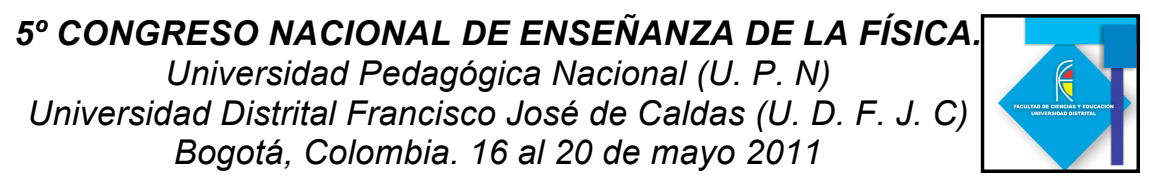

puede pensar que la matematización esté supeditada a los niveles

particulares de entendimiento de las personas, pues en los procesos de formalización, la matematización misma es un proceso que se refina, se mejora con la intencionalidad de explicar un fenómeno. En este sentido, la enseñanza de la física debe crear condiciones que permitan en los sujetos el mejoramiento continuo de las abstracciones que viabilicen la matematización de los fenómenos.

Sobre las representaciones que se exhiben en el clásico Óptica de Newton, el estudio histórico epistemológico permite resignificar las formas particulares que adquieren ciertos fenómenos como la descomposición de la luz en el espectro de colores. La lectura de estas representaciones permite valorar su simplicidad, y no por ello carencia de significado, en las posibilidades de ser enseñadas en el aula de clase. Un trabajo de representación permite conjeturar posibles relaciones entre los atributos de un objeto, Newton da muestra de ello. Esto sugiere en la enseñanza de las ciencias promover el uso de la representación en los estudiantes y maestros, en ese sentido, se debe evaluar la comprensión que tienen de sus elaboraciones para mejorar las formas de representación como un modo de significar los fenómenos.

Algunas acciones pueden favorecer los procesos de formalización y dependen de la forma en que se promueven, entre estas se tienen: medir, manipular, diseñar métodos de medición, confrontar resultados e ideas, reflexionar, entre otras. Dichas acciones poseen un sentido pedagógico en la orientación de los procesos de formalización de los fenómenos, ya que pueden ser objeto de evaluación de la comprensión de un fenómeno. Las acciones pueden fomentarse y desarrollarse en aquellos que, en su intento por interpretar el entorno, piensan y dan forma a los fenómenos físicos.

La enseñanza de las ciencias debe favorecer los procesos de formalización desarrollados por estudiantes y docentes mediante la implementación de unos nuevos objetivos pedagógicos. Estos objetivos obedecen a las teorizaciones adelantadas al respecto de la formalización de los fenómenos realizadas en esta investigación. Es decir, de los componentes involucrados, estos a la vez se pueden propiciar por razón de las acciones susceptibles de ser evaluadas por el maestro. El maestro de ciencias posee así una meta más en la enseñanza: formar personas partícipes en la construcción del conocimiento científico, autónomas en sus formas de comprender y reconocer los fenómenos pero conscientes del importante papel que desempeñan los otros en ese proceso.

\section{Referencias bibliográficas}

AYALA. M. Y et ál. (2008). Los procesos de formalización y el papel de la experiencia en la construcción del conocimiento sobre los fenómenos físicos. Editorial Universidad de Antioquia.

ROMERO, A. La formalización de los conceptos físicos. El caso de la velocidad instantánea. En: Revista Educación y Pedagogía. Medellín: Universidad de Antioquia, Facultad de Educación. Vol. XV, No. 35, (enero-abril), 2003.

PIERRRE, D. La teoría física, su objeto y su estructura. Cantidad y cualidad. 


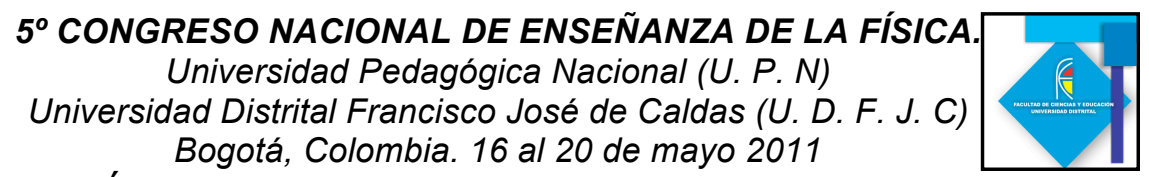

NEWTON, I. (1945). Óptica o tratado de las reflexiones, refracciones, inflexiones y colores de la luz. Traducción: Eugenio Días Del Castillo. Editorial Maestros de la Ciencia.

MARQUINA, J. (2003). La metodología de Newton. Universidad autónoma de México. En línea. Citado el 13 de julio de 2010.

SHAPIRO, A. (2006). La "filosofía experimental" de Newton. Universidad de Minnesota. En línea. Citado el 20 de junio de 2010. ashapiro@physics.umn.edu

MATTHEWS, M. R. Historia, Filosofía y Enseñanza de las ciencias: La aproximación actual. En: Enseñanza de las Ciencias. 1994.

STAKE, R. E. (1998). Paradigmas cualitativos y cuantitativos. En: Investigación como estudio de casos. Madrid: Ediciones Morata. 